\title{
Climate, mathematics, and the Canary Islands
}

\author{
José M. Pacheco* and N. Hayek** \\ * Departamento de Matemáticas \\ Universidad de Las Palmas de Gran Canaria \\ Campus de Tafira Baja \\ 35017 Las Palmas, Spain \\ ** Universidad de La Laguna /Academia Canaria de Ciencias. \\ La Laguna (Tenerife), Spain
}

\section{Introduction.}

It is difficult to provide a simple definition of climate. A vague idea, matching the intuitive notion of the layman, is the following: Climate is an average state of the atmospheric weather. Such a definition must be made precise enough in order to establish the ranges of spatial and temporal variability involved in it for a scientific study of the problem (Figure 1).

Four main concepts are taken into account in order to understand what climate is:

a) A notion of temporal durability. Thus, climate can be considered as some expected and characteristic feature of the spatial domains under consideration. Therefore, climate modelling has in some sense a more diagnostic character than weather studies, where prognostic features are predominant.

b) The just cited spatial domains are defined, as a rule, in a loose way; so boundary conditions incorporated in models are difficult to define and to formulate. The mathematical framework is that of free boundary problems. Land topography must be included among the limiting conditions in climatic studies.

c) The mathematical description of climate is achieved through the analysis of some dynamical spatio-temporal equilibrium in the values of the climatic variables.

d) A statistical interpretation of the three above points: This is the basis of any climatic classification.

Perturbations in the perception of any of these four concepts is generically known as climate change, and thus introduces a general philosophy on how to perform climate change experiments.

Global climate and climate change modelling has such a degree of complexity that the task lies beyond the limits of today's Mathematics and Computer Science (Lions,1991). Therefore, partial approaches are needed, focusing mainly on the following points:

a) To identify and to understand in depth the various physical processes involved between the different fluid envelopes of the Earth (Feistel, 1993). These include not only the atmosphere, but also the world ocean and ice caps, as well as their vertical stratifications.

b) Adequate mathematical modelling for the physical processes, depending on the underlying physics and the appropriate scaling of the phenomena under consideration (Giorgi, 1995; Liu and Yang, 1994; Lorenz, 1969; North, 1993; Vannitsem and Nicolis, 1994). 
c) Development of new mathematical and computational techniques, not only in the field of numerical analysis, but also in the realm of data acquisition and treatment. This includes not only new statistical tools: Expert system modelling is also an emerging alternative (Cheeseman and Oldford, 1994). Stochastic modelling is also coming into age with interesting applications to climate science (Wiesenfeld and Moss, 1995).

First of all, a physical definition of climate will be presented. Later on, problems in the mathematical description will be considered (Haltiner 1970, Pedlosky 1987).

Physically speaking, climate can be defined as some spatio-temporal average of the energy budget determined by the global mechanisms that transport, distribute and store the incoming Solar energy. Therefore, the "natural" approach to climate modelling is to formulate the so-called "energy balance models" (EBM) (Budyko, 1969; Sellers, 1969). The contribution of vulcanism has sensible effects on certain observation scales in this budget. Moreover, anthropic effects must be taken into account, for man-made energy manipulation can easily interfere with the natural cycles (Herring and Hobbs, 1994). As a consequence, the idea of control must be seriously incorporated in climatic studies.

Climate modelling is achieved though the selection of some descriptors, usually called state variables. These are macroscopic quantities whose evolution in time and space is observed from a theoretical viewpoint. A catalogue of these variables follows:

a) In the case of short and medium range studies, air temperature, sea surface temperature (SST), water vapor in the atmosphere and salinity of the sea water, as well as $\mathrm{CO}_{2}$ and $\mathrm{O}_{3}$ concentrations, are the most usual state variables in addition to wind, marine current and pressure fields. In this case climate modelling is best achieved though some variations of general circulation models (GCM). Tradition in climatic studies analyzes their evolution in 30year long cycles, though the tendency exists to consider shorter time intervals, even to the limit of deterministic weather prediction, in order to include sharper and finer variations in the realm of climate science.

b) In the case of long range studies, as is the case of paleoclimatic ones, the following are more adequate variables: Temperature at the thermocline bottom and various traces of dissolved or trapped gases (e.g. the ratio $\left[\mathrm{O}_{18}\right] /\left[\mathrm{O}_{16}\right]$ )in ice records. This is a consequence of some considerations to be made later on.

The evolution of the state variable values is described by the set of physical laws they obey to (Bjerknes, 1904). The mathematization of these laws is often difficult to separate from their formulation itself (Haltiner, 1970). In any case, three sets of problems are found:

a) The choice of the right Mathematics and the formulation of basic results. Problems in this group include existence and uniqueness results for various classes of equations, several types of estimates reflecting the physical soundness of the hypotheses, etc. (Diaz and Lions, 1993).

b) Computational questions arise in a natural way, given the relative scarcity of analytic solutions (Díaz and Lions, 1993; Hernández, Pacheco and García, 1983a; Lions, 1991) for most sets of equations as soon as a minimal complexity is reached. On the other hand, computational power is increasing in such a way that some problems can be viewed in a different way. As an example, rounding error analyses, with a 64-bit arithmetic could yield place to problems of storage and data retrieval of large mathematical structures.

c) Spatial and temporal limits of the system and boundary conditions deduced from them.

A climatic study of the Canary Islands from the modelling point of view starts with the difficulty of determining the system limits: The Archipelago lies in a very complicated interface (Figure 2) between the Açores anticyclonic gyre dominating the Central Atlantic Ocean and the huge continental mass of the Saharian Desert. The main observable features are: the cold 
current of the Canaries, the predominance of the Trade Winds, the West African upwelling and the suspended dust (Schutz, 1979). These are the true elements of the Canary Islands climate.

\section{Complex Systems and Mathematics.}

A complex system is one where many different spatial and temporal scales are needed for an accurate description of its behavior. Climate is indeed a complex system: Temporal scales range from the infinitesimal time neded for a phase change to the thousands of years of residence time of sea water in the deep Ocean basins. On the other hand, spatial scales vary from the few $\mu \mathrm{m}$ of the incoming short wave Solar radiation to the $10^{7} \mathrm{~m}$ scale of the Rossby and Kelvin waves involved in the El Niño-Southern Oscillation phenomenon. It is a wise advice to distinguish three groups of variables according to their temporal scales (Morantine and Watts, 1994; Watts et al. 1994):

a) The "natural" scales of the phenomenon are those of the macroscopic variables selected by the observer for a description of the system status. Climate can be described, as was pointed out above, by the same variables employed in models of the atmosphere, supplemented with those describing tsea-air interaction. As a result, a climate model is the coupling of an atmospheric GCM and a marine one, each one with its ordinary natural scales (Figure 3 ). Ice caps are included in the marine part of the model.

b) The "slow" variables are those that can be considered as constants when compared with the state variables. Their evolution can not be observed with the resolution accorded to the space variables. As a rule, they represent boundary conditions or physical constants and can be considered as parameters. In climate studies most lithospheric topographic constraints belong to this type, as well as the geological timescale. The detection of "truly slow" variables is one of the main concerns of the climate study (Grassberger,1986; Lorenz, 1986; Temam, 1995)). Other physical barriers, like the tropopause, the thermocline, etc., may belong or not to this class depending on the problem considered.

c) "Fast" variables are those whose fluctuation with respect to the state variables is detected as noise by the observer. Variables belonging to this class could be eliminated from the system if their influence is proved to be irrelevant. This not a simple problem: It is related with the ergodicity or not of the weather-climate system and is an open field of study (Fraedrich and Ziehmann-Schlumbohm,1994).

The above classification allows the modeller to concentrate on the selection and behavior of a number of variables notably smaller than that of all interesting ones, as was already pointed in the Introduction.

Scale considerations are most relevant when translating a mathematical model into a computational one. The discretization step introduces a non physical, spurious parameter, i.e. the mesh size of the computational grid (to be precise, a vector mesh size concept must be provided) (Adams et al., 1993). Therefore the output of a computational climate model can yield physically senseless results because of the unresolved features of subgrid scale. A well known example are climate change experiments: These can show extra heat sources not consistent with the Physics when run under the hypothesis that $\mathrm{CO}_{2}$ concentration is doubled. This problem is an interesting one within Bifurcation Theory.

The Canary Islands climate system, which must be studied at "regional" scales, is a typical case of nearly-subgrid system. The usual climate models employ a rectangular grid with a linear mesh around $300 \mathrm{~km}$, in such a way that four adjacent rectangles would cover the entire Archipelago, and any island lies completely within one single rectangle. Vertical discretizations with 10 to 20 atmospheric levels are not fine enough to resolve many of the small scale features which determine the Islands climate. 
As a rule, climatic records show a repetitive basic pattern upon which apparently random fluctuations are observed which do not modify the overall structure. This structure is usually known as an "attractor", which is roughly equivalent to the existence of a slow scale variable. There exist algorithms to identify such structures from time series records (Grassberger and Procaccia, 1983; Short, 1993). Therefore, a new non classical modelling is available. The idea behind the attractor reconstruction implies embedding of the time series in an adequate $n$-dimensional vector space, where similar -in a way to be precisely definedfragments of $n$ consecutive terms build sets of close vectors. Predictive capabilities of the reconstructed attractor depend on its fractal dimension and shortcomings of the method appear if the time series is not long enough.

In the Canary Islands this study has not been done, though records of air temperatures and precipitation are kept for agricultural and trade reasons since the 1850 's. It must be noted here that problems related with water supplies are a constant in the culture of the Islanders. On the other hand, longer range studies are more difficult to do because of the lack of records originated by two main reasons: The first is overexploitation of the aquifers that has changed a large amount of fossile waters (more than 5000 years) into brackish water due to marine intrusions and to local chemical interactions thus loosing an invaluable climatic record. The second is systematic deforestation, which is the cause that tree-ring analyses are impossible due to the lack of old enough trees in most of the Islands.

\section{The Climatic cycle.}

The energy cycle in the Earth starts with the incoming shortwave (less than $4 \mu \mathrm{m}$ wavelength) Solar radiation. Atmospheric water and water vapor are transparent to it, as well as the first few centimeters of seawater. A substantial part of the Solar energy reaching the sea surface is stored in this thin layer (Pelegrí et al., 1995; Stephens et al., 1993). Radiation reaching the continental land mass comes back to the atmosphere, for the calorific capacity of land materials is quite low. A part of this reflected radiation, now of a longer wavelength, remains trapped under the cloud cover: This is called the greenhouse effect. There are also local anthropic effects contributing to the conservation of heat in the lower atmosphere, the so called heat island effects. $\mathrm{CO}_{2}$ concentration in the atmosphere is closely related with radiative properties, so this is a basic variable in climate change experiments.

The role of ocean dynamics is a basic one in the global distribution of heat (Bindoff and McDougal, 1994; Drijfhout, 1994), for sea water has a heat capacity some three orders over that of the land. Heat transport has two main mechanisms:

a) Within the sea, currents originated by temperature and salinity gradients. These are responsible of the origin of North Atlantic polar water, which is later on distributed along the world ocean by a kind of global conveyor belt (Hughes and Weaver, 1994; Weaver and Hughes, 1994) (Figure 4).

b) Within the atmosphere, the release of latent heat is the main cause of heat transfer to the upper atmosphere through the mechanisms of evaporation and precipitation (Hernández, Pacheco and García, 1983b and 1987; Zeng and Pielke, 1993).

The cycle is closed by the mechanic energy transfer from the atmosphere to the sea surface via wind stresses (Charney, 1955), giving rise to the wave system and to some types of surface currents. Winds are generated by temperature and density gradients in the atmosphere, and a continuous feedback is achieved between the two parts of the climate system (Pereira and Mascarenhas, 1994). A history of this mechanical interaction is stored in the finer structures of the thermocline (Liu and Pedlosky, 1994), below which atmospheric interaction is negligible. No good measurements of the amount of mechanical energy transferred to the sea surface are known, only rough estimates with great variability. Thus, the choice of the bottom 
temperature of the thermocline is justified in long scale climate experiments if episodes with time scales less than a few decades are to be filtered out.

Short time predictability of the atmospheric weather is simply a reflection of the fact that the atmosphere stratification has a very short turnover time. On the contrary, turnover times for the ocean waters are acknowledged to be of the order of hundreds or thousands of years: A well known case is the Black Sea. Thus the stratification of the water column keeps a record of climate fluctuations, because the cold water generated in cold climate episodes sinks down rather quickly to the bottom, but during hot episodes warm surface waters can not transfer heat to the bottom.

The above discussion shows that SST is the main boundary constraint in atmospheric GCM's. In other words, it can be shown that the air temperature is forced by and follows the slower oscillations of the SST, thus providing an argument for the implementation of "marine only" climate models (Hughes and Weaver, 1994; Weaver and Hughes, 1994).

The elimination of atmospheric dynamics in climate models could only be achieved if the atmosphere would prove to be an ergodic system. Ergodicity of the atmosphere is an open problem (Feistel,1993; Nagai et al., 1992) and it is only in the last years that large-scale experiments are starting their way. Theoretical modelling is still far from today's state of the art.

In the Canary Islands several studies are in due course to identify the local mechanisms of heat storage and transport in the Eastern-Central Atlantic Ocean area (Pelegri et al., 1995). The conclusions show quite surprisingly that there is a seasonal heat accumulation well under the sea surface, a fact that could be interpreted as a signal of a slow manifold. On the other hand, the Canaries water column has been systematically explored in several oceanographic campaigns in the years 1957, 1981 and 1992, but no climate studies have been carried on with this data base.

\section{On Climate Modelling.}

It was pointed out in the Introduction that climate modelling has a predominantly diagnostic character. Nevertheless, prognostic studies can also be envisaged with the aim of making short and medium term forecasts, in order to assess the expected tendencies: This is but one way of understanding the structure of climatic changes.

A global climatic model expresses some kind of energy budget. If an average temperature $u(x, t)$ is employed as the main descriptor, the already classical Budyko-Sellers energy-balance model (with $x=\sin \phi, \phi=$ latitude) for the closed interval $[-1,1]$ contans the nonlinear parabolic equation:

$$
\frac{\partial u}{\partial t}-\frac{\partial}{\partial x}\left[D\left(x, \frac{\partial u}{\partial x}\right) \frac{\partial u}{\partial x}\right]=R_{a}-R_{e}
$$

and appropriate free boundary conditions.

More local models, on the other hand, tend to be more complicated. It is now time to recall that the main features in the dynamics of geophysical fluids are captured in the so-called Navier Stokes system: First of all, a set of equations with the same structure is considered. These are models for the time-space evolution of several different magnitudes $\boldsymbol{X}$, either vector or scalar ones. Among them there are the flux components (wind, marine currents), temperatures, water vapor and salt concentrations (atmosphere or sea), various pollutant 
concentrations, etc. All of them show the following structure, where $v$ is the flux velocity vector:

$$
\begin{gathered}
\frac{\partial X}{\partial t}=\text { large scale terms (first and second) }+ \\
+ \text { small scale terms (third and fourth) }= \\
-v \cdot \text { gradX }+ \text { Gradient dependent term }+ \\
\text { Diffusion term + Interaction and sources term. }
\end{gathered}
$$

For instance, in the atmospheric part, when $\boldsymbol{X}=v$, a system is obtained yielding a mahematical form of Newton's Second Law, or momentum equations:

$$
\frac{\partial v}{\partial t}=-v \cdot g r a d v-F(\operatorname{grad} p, \Omega)-\alpha D v+f
$$

where $\boldsymbol{p}$ is pressure, $\boldsymbol{\Omega}$ is the vector speed of the rotating Earth and $\alpha$ is a diffusion coefficient. If $v$ is changed by temperature, $\boldsymbol{T}$, an analogous equation is obtained, where the second term models adiabatic vertical movements of air parcels, etc. Moreover, a continuity or conservation equation must be added, as well as a state equation, usually the hydrostatic equilibrium equation.

In practice several simplifying assumptions are made that give rise to different versions of the Navier Stokes system. The main feature are nonlinearity -in principle, due to the advective term $v \cdot g r a d X$ - and stiffness (because of the many scales involved) in the computational study.

In addition to classical computational problems, predictability analyses (Nicolis, 1990; ECMWRF, 1988) are of foremost importance: The Navier Stokes system, when employed in an instantaneous description of weather, is sensible to changes in the supplementary conditions and a dissipative one. Thus it is a chaotic system with a limited predictive ability: The deterministic predictive horizon of some ten days. In order to obtain forecasts for longer periods, the model equations must be modified. The idea is to include some averaging procedure over variations in the supplementary conditions. Nevertheless, averaging is a linear operation and does not commute with the nonlinear terms. Thus, climate models differ from GCM's in the way they deal with the nonlinear terms.

\section{Which climate model? The Canary Islands case.}

GCM based numerical climate models are not adequate for small areas, for most features belong to subgrid scales. Thus a considerable part of the relevant information is dealt with as noise or is generically incorporated into the small scale terms.

In the Canary Islands case, such a model would yield a description of climate closer to the travel agency stereotype than to the actual set of highly dispersed microclimatic areas. Therefore, the Canaries illustrate a typical case where ad hoc modelling is needed.

Along the preceding paragraphs, several observations have been made on how general theoretical principles can be restricted to this case. A brief summary is the following:

a) A large data base is needed in order to establish boundary conditions over The Saharian Desert and the Central Norh Atlantic. In spite of active research in both fields with the help of satellite imagery, existent records are fragmentary. 
b) The synoptic scale, a vague expression meaning a spatial size somewhere between 1000 and $1500 \mathrm{~km}$, when combined with the usual grid meshes, cannot resolve the main features of the Islands climate.

c) There is a considerable lack of paleoclimatic fossile records and available data about the water column have not been climatically exploited.

Therefore, the following alternatives seem most feasible:

a) To carry on finer observational results on geophysical magnitudes, based both in satellite and in situ measurements, with massive Statistical information processing in order to obtain accurate descriptions. This idea could yield a local energy-balance model theory in order to explain some cyclic behaviours.

b) To incorporate adequate parameterizations for subgrid scale features in GCM based models. This is an open problem, whose difficulty depends either on the existence of an acceptable theoretical basis or the availability of solely empirical evidences. As an important question, it must be remarked here that radiative properties in the Islands atmosphere depend with regularity on the distribution and fractal properties of Saharian dust. Only partial approaches to this problem are available.

c) Ad hoc model building via local versions with much finer grid meshes and an adequate choice of the state variables. These "nested models" could live as submodels of some GCM's and would incorporate the results of a). This seems to be the most appropriate choice with today's state of the art.

d) Data analysis, both of recent and paleoclimatic records, not only from a multivariate statistical viewpoint, but including more sophisticated tools. Data base analyses and reconstruction of air parcel trajectories is but an example of immediate applicability (Sancho et al., 1992). Expert system modelling, as well, is a field of growing interest due to the availabillity of computational resources. This approach, which attempts to conjecture and to explain possible causal relationships, needs the development of new computational tools. In a not so far future this seems to be a most interesting and practical alternative to today's physically based mathematical modelling techniques.

\section{References}

Adams E et al. (1993) Computational chaos may be due to a single local error, J. Comput. Phys, $104,241-250$.

Bindoff N, McDougal T (1994) Diagnosing climate change and ocean ventilation using hydrographic data, J. Phys. Ocean., 24-6, 1137-1152.

Bjerknes V (1904) Das Probem der Wettervorhersage betrachtet vom Standpunkt der Mechanik und der Physik, Met. Zeit., 21, 1-6.

Budyko M (1969) The effects of solar radiation variations on the climate of the Earth, Tellus, 21, 611-619.

Charney J (1955) The generation of ocean currents by the wind, J. Marine Res., 14, 477-498.

Cheeseman P, Oldford R, eds. (1994) Selecting models from data (Artificial Intelligence and Statistics, IV), Lecture Notes in Statistics, 89, Springer Verlag, Berlin.

Díaz J I, Lions J L, eds. (1993) Mathematics, climate and the environment, Masson, Paris. Drijfhout S (1994) Heat transport by mesoscale eddies in an Oceanic Circulation Model, $J$. Phys. Ocean., 24-2, 353-369.

ECMWRF (1988) Predictability in the medium and extended range. Proceedings of a Workshop held in May 1988 at Reading, UK.

Feistel R (1993) Climate and Physics of Evolution, Nonlinear Processes in Geophysics, (to appear).

Fraedrich K, Ziehmann-Schlumbohm C (1994) Predictability experiments with persistence forecasts in a red-noise atmosphere, Q.J.R. Met. Soc.,120, 387-428.

Giorgi F (1995), These Proceedings.

Grassberger P (1986) Do climatic attractors exist?, Nature, 323, 609-612. 
Grassberger P, Procaccia I (1983) Measuring the strangeness of strange attractors, Physica D9, 189-195.

Haltiner J (1970) Numerical Weather Prediction, J. Wiley \& Sons, New York.

Hernández E, Pacheco J, García R (1987) Evidencias del desarrollo de ondas frontales en las inundaciones de Levante de 1982, Rev. R. Soc. Ci. Ex.Fís.Nat, LXXXI, 2, 299-314.

Hernández E, Pacheco J, García R (1983a) Métodos numéricos en la predicción del tiempo, Rev. de Geofísica, 39.2, 151-164.

Hernández E, Pacheco J, García R (1983b) Inclusión de la liberación de calor latente en las ecuaciones de un modelo de predicción, Communication to the $V$ Asamblea Nacional de Geofísica y Geodesia, Madrid.

Herring J, Hobbs P (1994) Radiatively driven dynamics of the plume from Kuwait 1991 oil fires, J. Geophys. Res., 99 D9, 18809-18826.

Hughes T, Weaver A (1994) Multiple equilibria of an asymmetric two-basin ocean model, $J$. Phys. Ocean., 24-3, 619-637.

Lions J L (1991) "ElplanetaTierra", Espasa Calpe, Madrid.

Liu Z, Pedlosky J (1994) Thermocline forced by annual and decadal surface temperature variation, J. Phys. Ocean., 24-3, 587-608.

Liu Z, Yang H (1994) The intergyre chaotic transport, J. Phys. Ocean., 24-8, 1768-1782.

Lorenz E (1969) The predictability of a flow which possesses many scales of motion, Tellus, 16(1), 289-307.

Lorenz E (1986) On the existence of a slow manifold, J. Atmos. Sci., 43(15), 1547-1557.

Morantine M, Watts R (1994)Timescales in energy balance climate models 2: The intermediate time solutions, J. Geophys. Res., 99 D2, 3643-3654.

Nagai T et al. (1992) El Niño-Southern Oscillation simulated in an MRI atmosphere-ocean coupled general circulation model, J. of Climate, 5-11, 1202-1233.

Nicolis C (1990) Chaotic dynamics, Markov processes and climate predictability, Tellus 42A, 401-412.

Pedlosky J (1987) Geophysical fluid dynamics, Springer Verlag, Berlin.

Pelegrí J L et al. (1995) These Proceedings.

Pereira C, Mascarenhas A (1994) Numerical simulation of an oceanic front response to an atmospheric frontal passage, J. Geophys. Res., 99 C8, 16081-16094.

Sancho P et al. (1992) A five-year climatology of back-trajectories from the Izaña baseline station, Tenerife, Canary Islands, Atmos. Envir., 26 A6, 1081-1096.

Schutz L (1979) Sahara dust transport over the North Atlantic Ocean: Model calculations and meaurements, in Morales C (ed.) Saharan dust: Mobilization, transport, deposition (267-278), J. Wiley \& Son, New York.

Sellers W (1969) A global climate model based on the energy balance of the earth-atmosphere system, J. Appl. Met., 8, 392-400.

Short K (1993) Direct calculation of metric entropy from time series, J. Comput. Phys., 104, 162-172.

Stephens G et al. (1993) The Earth's radiation budget and its relation to atmospheric hidrology, J. Geophys. Res., 98 D3, 4931-4950.

Temam R (1995) These Proceedings.

Vannitsem S, Nicolis C (1994) Predictability experiments on a simplified thermal convection model: The role of spatial scales, J. Geophys. Res., 99 D9, 10377-10386.

Watts R et al. (1994) Timescales in energy balance climate models 1: The limiting case solutions, J. Geophys. Res., 99 D2, 3631-3642.

Weaver A, Hughes T (1994) Rapid interglacial climate fluctuations driven by North Atlantic ocean circulation, Nature, $367,447-450$.

Wiesenfeld K, Moss F (1995) Stochastic resonance and the benefits of noise: from ice ages to crayfish and SQUIDs, Nature, 373, 33-36.

Zeng X, Pielke R (1993) Error-growth dynamics and the predictability of surface thermally $i$ nduced atmospheric flow, J. Atmos. Sci, 50-17, 2817-2844. 


\begin{tabular}{|l|l|}
\hline $10^{1} \mathrm{~s}$ (mins) \\
$10^{3} \mathrm{~s}$ (hours) \\
$10^{7} \mathrm{~s}$ (years) \\
$10^{7} \mathrm{~s}$ (years) \\
$10^{7} \mathrm{~s}$ (years) \\
$10^{9} \mathrm{~s}$ (century) \\
$10^{7}$ s(years)
\end{tabular}$\quad$\begin{tabular}{l}
$10^{3} \mathrm{~m}$ \\
$10^{4} \mathrm{~m}$ \\
$10^{5} \mathrm{~m}$ \\
$10^{6} \mathrm{~m}$ \\
$10^{7} \mathrm{~m}$ \\
$10^{7} \mathrm{~m}$ \\
$10^{7} \mathrm{~m}$ \\
\hline
\end{tabular}

Temporal
Spatial
Atmospheric turbulence

Atmospheric convection

Yearly seasons

Upper ocean mixing

ENSO

Global ocean circulation

CLIMATE

Figure 1

The Scales of the Climate System

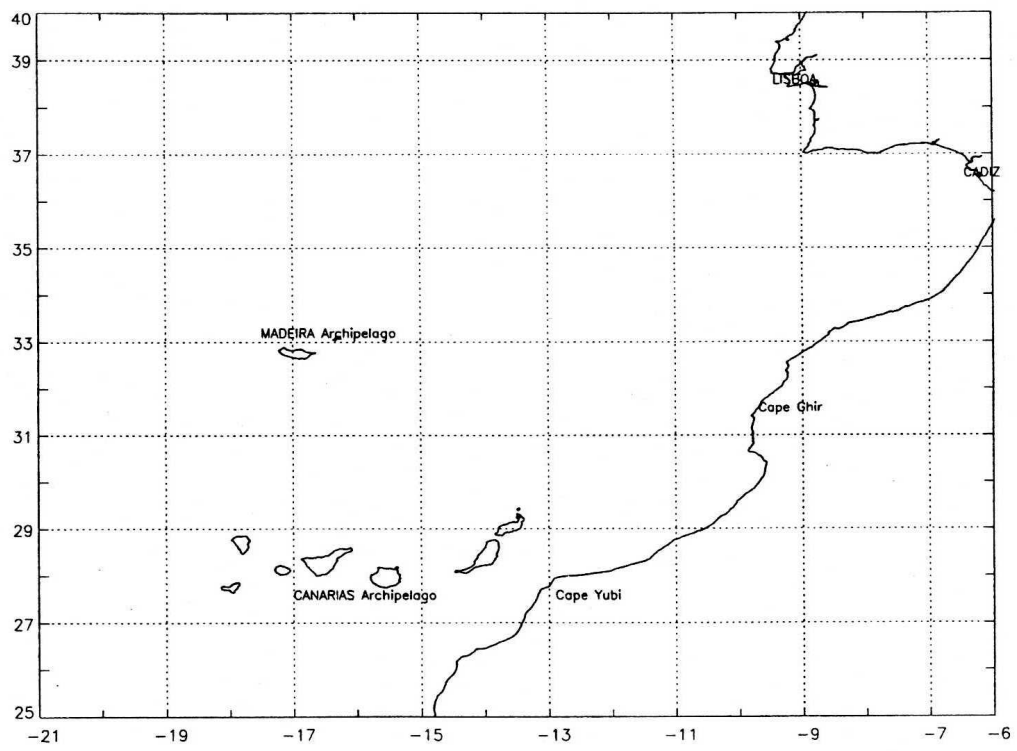

Figure 2

The Canary Islands 


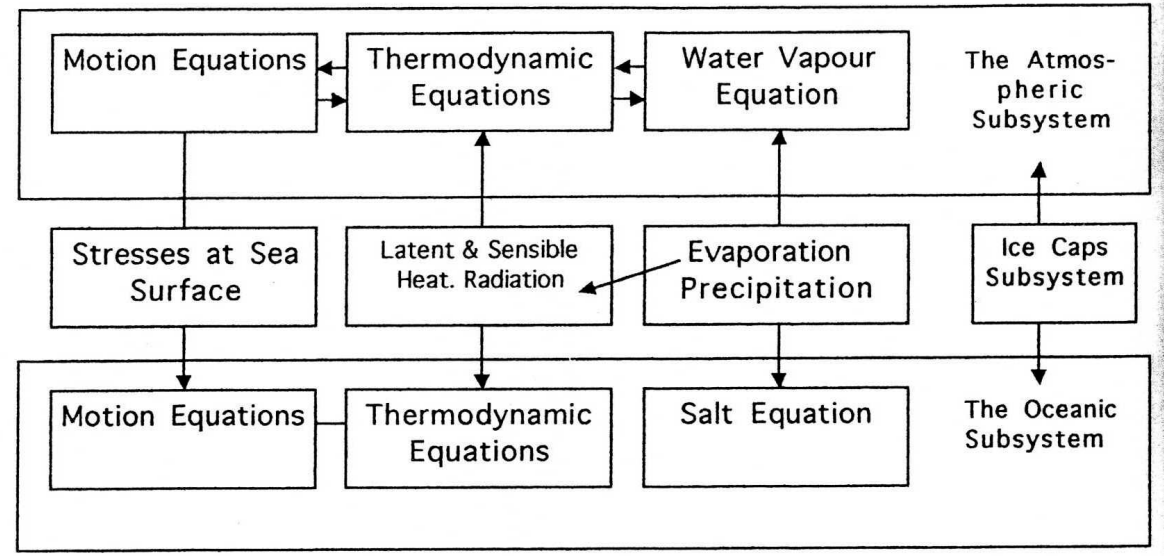

Figure 3

The Climate System

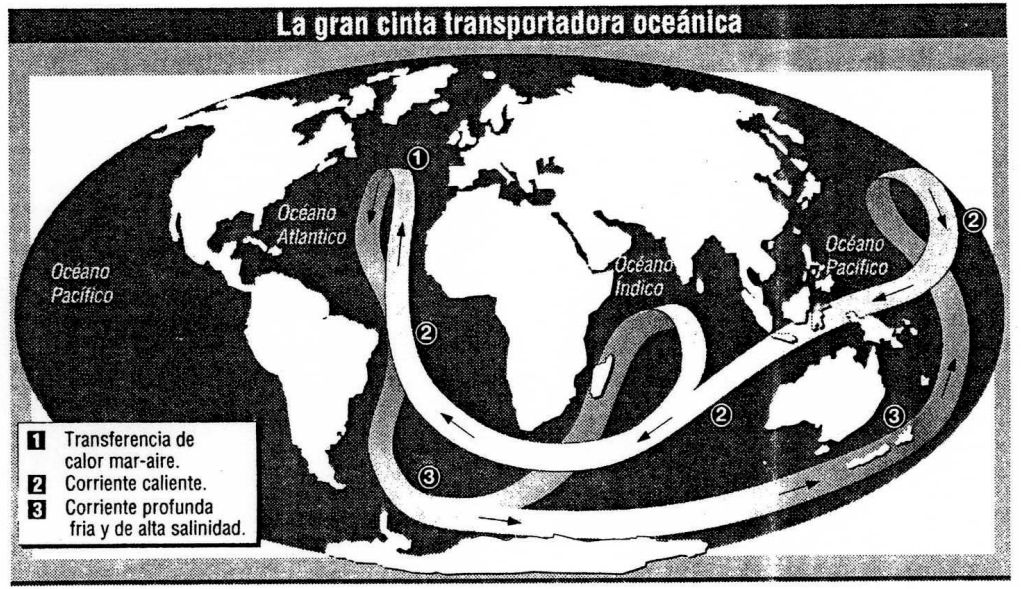

Figure 4

The Climate Conveyor Belt

(Taken from El País February 3rd, 1994) 